\title{
The Faulhaber Problem on Sums of Powers on Arithmetic Progressions Resolved
}

\author{
Do Tan $\mathrm{Si}^{1}$ \\ ${ }^{1}$ The HoChiMinh-city Physical Association, Vietnam \\ Correspondence: Do Tan Si, The HoChiMinh-city Physical Association, Vietnam. E-mail: tansi_do@yahoo.com
}

Received: January 22, 2018

Accepted: January 25, 2018

Online Published: March 15, 2018

doi:10.5539/apr.v10n2p5

URL: https://doi.org/10.5539/apr.v10n2p5

\begin{abstract}
We prove that all the Faulhaber coefficients of a sum of odd power of elements of an arithmetic progression may simply be calculated from only one of them which is easily calculable from two Bernoulli polynomials as so as from power sums of integers. This gives two simple formulae for calculating them. As for sums related to even powers, they may be calculated simply from those related to the nearest odd one's.
\end{abstract}

Keywords: Faulhaber conjecture, Power sums of integers, Power sums on arithmetic progressions, New properties of Bernoulli polynomials, Operator calculus

\section{Introduction}

The problem of showing the sum of $m^{\text {th }}$ power of integers, denoted in this work by $S_{m}(n)$, as a polynomial function of $n$ has fascinated mathematicians from the antiquity until nowadays as we can see for examples in a master thesis of Coen (1996), in Beery (2010), etc.

As for the problem of expressing $S_{m}(n)$ as a sum of powers of $S_{1}(n)$, Johann Faulhaber (1631) is certainly the most celebrated for his conjecture saying that a sum of odd power of integers are homogeneous polynomials in sum of them, appeared in a booklet entitled Academia Algebro published in 1631, rediscovered surprisingly only in 1981 by Edwards (1986) at Cambridge university. After Faulhaber, Jacobi (1834) proved that $S_{m}(n)$ may be calculated from $S_{1}(n)$ and $S_{2}(n)$. Long years passed until Edwards (1986) showed recently how to obtain the coefficients of powers of $S_{1}(n)$ in $S_{2 k+1}(n)$, called hereinafter Faulhaber coefficients, by matrix inversion, based on another proof given by Tits (1923). Ultimately Truth (1993) explored a computation-based approach by which Faulhaber may well have discovered such results, and solves a 360-year-old riddle that Faulhaber presented to his readers. Truth also showed that Faulhaber coefficients can moreover be generalized to non integer exponents.

More generally, about the problem of representing sums of $m^{\text {th }}$ power of the elements of an arithmetic progression $z, z+1, \ldots, z+n-1$ when $m$ is odd, denoted herein by $S_{2 k+1}(z, n)$, in term of polynomial in $n$ we have the formulae of Dattoli, Cesarano, Lorenzutta (2002) and of Do (Do T.S.2017b). As for the Faulhaber problem of representing $S_{2 k+1}(z, n)$ as homogeneous polynomial in $S_{1}(z, n)$, we notice the very recent publication of Chen,

$\mathrm{Fu}$, Zhang (2009). These authors utilized the Dattoli, Cesarano, Lorenzutta formula that links $S_{2 k+1}(z, n)$ to the Bernoulli polynomials $B_{m}(z+n)$, the addition theorem for Bernoulli polynomials and the property $B_{2 k+1}(1 / 2)=0$ in order to prove the Faulhaber conjecture concerning $S_{2 k+1}(z, n)$. The formula illustrated it may be utilized to calculate the coefficients of powers of $S_{1}(z, n)$, i.e. the Faulhaber coefficients. Nevertheless we see that the practical calculations are cumbersome.

Continuing the researches on Faulhaber conjecture on power sums on arithmetic progressions we propose in this work firstly to improve by simplification the quoted proof given by Chen, Yang and Zhang; secondly to obtain a simple method for calculating the Faulhaber coefficients related to sums of odd powers of integers $S_{2 k+1}(n)$; thirdly to obtain those related to power sums on arithmetic progressions $S_{2 k+1}(z, n)$ and $S_{2 k}(z, n)$.

It is found that all the Faulhaber coefficients of $S_{2 k+1}(z, n)$ may simply be calculated from one of them which is equal to $B_{2 k+1}(z) B_{1}^{-1}(z)$ as so as from $S_{2 k+1}(z)$. This gives rise to two simple formulae for calculating $S_{2 k+1}(z, n)$. As for sums related to even powers $S_{2 k}(z, n)$, we will see that they may be calculated simply from $S_{2 k+1}(z)$. 


\section{Faulhaber Formula for Sums of Powers on Arithmetic Progressions}

2.1 In a Recent Paper (Do, 2017b) on Sums of Powers of Elements of an Arithmetic Progression

$$
S_{m}(z, n)=z^{m}+(z+1)^{m}+\ldots+(z+(n-1))^{m}
$$

we have proven by utilizing translation operators the property

$$
S_{m}(z, n)=\frac{e^{n D_{z}}-1}{e^{D_{z}}-1} z^{m}
$$

from which one get easily an exponential generating function. Comparison of it with the generating function of Bernoulli polynomials leads to the formula

$$
(m+1) S_{m}(z, n)=\sum_{k=1}^{m+1}\left(\begin{array}{c}
m+1 \\
k
\end{array}\right) B_{m-k+1}(z) n^{k}:=(B(z)+n)^{m+1}-B^{m+1}(z)
$$

where in the right hand side the undefined symbol $B^{k}(z)$ is to be replaced with the Bernoulli polynomial $B_{k}(z)$.

From (3) and the symbolic relation

$$
B_{j}(z):=(B+z)^{j}
$$

we get

$$
(B(z)+n)^{m+1}:=((B+z)+n)^{m+1}:=(B+(z+n))^{m+1}
$$

which proves directly the formula of Dattoli, Cesarano, Lorenzutta (2002)

$$
(m+1) S_{m}(z, n)=(B+z+n)^{m+1}-(B+z)^{m+1}=B_{m+1}(z+n)-B_{m+1}(z)
$$

Now from the well known propety

$$
B^{\prime}{ }_{m+1}(z)=(m+1) B_{m}(z)
$$

we get

$$
\int_{0}^{n} B_{m}(t+z) d t=\frac{1}{m+1} \int_{0}^{n} \partial_{t} B_{m+1}(t+z) d t=\frac{1}{m+1}\left(B_{m+1}(n+z)-B_{m+1}(z)\right)
$$

which by (5) leads to the elegant formulae

$$
\begin{gathered}
S_{m+1}(n)=\int_{0}^{n} B_{m}(t) d t:=\int_{0}^{n}(B+t)^{m} d t \\
S_{m+1}(z, n)=\int_{0}^{n} B_{m}(z+t) d t:=\int_{0}^{n}(B+z+t)^{m} d t
\end{gathered}
$$

\subsection{Proof of the Faulhaber Conjecture}

Following the ideas of Chen, Fu and Yang (2009) we utilize the addition formula to get the expansion

$$
B_{m}(z+n)=B_{m}\left(z-\frac{1}{2}+n+\frac{1}{2}\right)=\sum_{k=0}^{m}\left(\begin{array}{l}
m \\
k
\end{array}\right) B_{m-k}\left(\frac{1}{2}\right)\left(z-\frac{1}{2}+n\right)^{k}
$$

which by (5) and because $B_{k}(1 / 2)=\left(2^{-k+1}-1\right) B_{k}$ leads to

$$
2 m S_{2 m-1}(z, n)=\sum_{i=0}^{m}\left(\begin{array}{c}
2 m \\
2 i
\end{array}\right) B_{2 m-2 i}\left(\frac{1}{2}\right)\left(z-\frac{1}{2}+n\right)^{2 i}-B_{2 m}(z)
$$

But

$$
\left(z-\frac{1}{2}+n\right)^{2}=2\left(z-\frac{1}{2}\right) n+n^{2}+\left(z-\frac{1}{2}\right)^{2}=2 S_{1}(z, n)+\left(z-\frac{1}{2}\right)^{2}
$$

so that 


$$
\begin{gathered}
2 m S_{2 m-1}(z, n)=\sum_{j=0}^{m}\left(\begin{array}{c}
2 m \\
2 j
\end{array}\right) B_{2 m-2 j}\left(\frac{1}{2}\right)\left(2 S_{1}(z, n)+B_{1}^{2}(z)\right)^{j}-B_{2 m}(z) \\
S_{2 m-1}(z, 0)=0=\sum_{i=0}^{m}\left(\begin{array}{c}
2 m \\
2 i
\end{array}\right) B_{2 m-2 i}\left(\frac{1}{2}\right) B_{1}(z)^{2 i}-B_{2 m}(z)
\end{gathered}
$$

Combining these formulae we get finally

$$
2 m S_{2 m-1}(z, n)=\sum_{j=1}^{m}\left(\begin{array}{l}
2 m \\
2 j
\end{array}\right) B_{2 m-2 j}\left(\frac{1}{2}\right)\left[\left(2 S_{1}(z, n)+B_{1}^{2}(z)\right)^{j}-B_{1}^{2 j}(z)\right]
$$

and may pronounce

- The Faulhaber theorem:

$$
\text { " } S_{2 m-1}(z, n) \text { is a homogeneous polynomial of order } m \text { in } S_{1}(z, n) \text { " }
$$

together with

- The Chen, Fu and Yang theorem that we may condense into a concrete phrase:

$$
\text { “ }(2 k+2) S_{2 k+1}(z, n) \text { is equal to } \sum_{j=1}^{k+1}\left(\begin{array}{c}
2 k+2 \\
2 j
\end{array}\right) B_{2 k+2-2 j}\left(\frac{1}{2}\right)\left(2 S_{1}(z, n)+B_{1}^{2}(z)\right)^{j}
$$

without terms independent with respect to $n$ "

We note that as $B_{2 m}\left(\frac{1}{2}\right) \neq 0$, we don't have the property $(8)$ for $S_{2 k}(z, n)$.

For example, for $k=2$, from

$$
\sum_{j=1}^{3}\left(\begin{array}{c}
6 \\
2 j
\end{array}\right) B_{6-2 j}\left(\frac{1}{2}\right)\left(2 \lambda+B_{1}^{2}(z)\right)^{j}=\frac{7}{16}\left(2 \lambda+B_{1}^{2}(z)\right)-\frac{15}{12}\left(2 \lambda+B_{1}^{2}(z)\right)^{2}+\left(2 \lambda+B_{1}^{2}(z)\right)^{3}
$$

where $\lambda=S_{1}(z, n)$, we get by omitting the terms constant with respect to $n$

$$
6 S_{5}(z, n)=8 \lambda^{3}+\left(12 z^{2}-12 z-2\right) \lambda^{2}+\left(z^{2}-z\right)\left(6 z^{2}-6 z-2\right) \lambda
$$

Although from (11) we may calculate $S_{2 k+1}(z, n)$ as a homogeneous polynomial of $\lambda$, nevertheless we see that the calculations of the coefficients of $\lambda^{i}=S_{1}^{i}(z, n)$ are not so simple for high values of $k$.

For this reason in the following paragraphs we will try to obtain a simpler method for obtaining these coefficients, beginning with those of power sums of integers.

\section{Obtaining $S_{2 k+1}(n)$ as Sum of Powers of $S_{1}(n)$}

\subsection{Relations Between Coefficients of Powers of $S_{1}(n)$ in $S_{2 k+1}(n)$}

From the Faulhaber conjecture now verified we may write

$$
\begin{aligned}
S_{2 k+1}(n) & =a_{k, 1} S_{1}(n)+a_{k, 2} S_{1}^{2}(n)+\ldots+a_{k, k+1} S_{1}^{k+1}(n) \\
& =\sum_{j=1}^{k+1} \bar{a}_{k, j}\left(n^{2}-n\right)^{j} \quad \forall n,
\end{aligned}
$$

where

$$
\bar{a}_{k, j}=2^{-j} a_{k, j}
$$

so that to obtain $S_{2 k+1}(n)$ it suffices to calculate the Faulhaber coefficients $\bar{a}_{k, 1}, \bar{a}_{k, 2}, \bar{a}_{k, 3}, \ldots, \bar{a}_{k, k}, \bar{a}_{k, k+1}$

This may happily be done by comparing (12) with the formula giving $S_{2 k+1}(n)$

from the set of Bernoulli numbers $B_{l}$ as followed one may find in (Bernoulli, 1713)

$$
S_{2 k+1}(n)=\sum_{j=0}^{2 k+1} \frac{1}{2 k+2}\left(\begin{array}{c}
2 k+2 \\
j+1
\end{array}\right) B_{2 k+1-j} n^{j+1} \quad \forall n
$$

that we may put under the form 


$$
S_{2 k+1}(z)=\int_{0}^{z} B_{2 k+1}(t) d t=\frac{1}{2 k+2}\left(B_{2 k+2}(z)-B_{2 k+2}\right)
$$

or, alternatively, the Pascal formula (Beery, 2010 ) that we have put under the form

$$
S_{2 k+1}(n)=\sum_{j=1}^{2 k+1} P_{2 k+1 j}^{-1} n^{j+1}+B_{2 k+1} n
$$

where $P_{m \mathrm{j}}^{-1}$ are elements of the inverse of the Pascal matrix

$$
P_{m j}=\left(\begin{array}{c}
i+1 \\
j
\end{array}\right)
$$

which may be calculated by a simple algorithm and tabulated until $P_{1717}^{-1}$ (Do, 2017a).

From (14) and (15) we have the relation

that we will extend with

$$
\begin{gathered}
B_{m}=-\sum_{j=1}^{m} P_{m \mathrm{j}}^{-1} \\
P_{m \mathrm{j}}^{-1}=\frac{1}{j+1}\left(\begin{array}{c}
m \\
j
\end{array}\right) B_{m-j}=\frac{1}{m+1}\left(\begin{array}{c}
m+1 \\
j+1
\end{array}\right) B_{m-j}
\end{gathered}
$$

$$
P_{m 0}^{-1}=\frac{1}{0+1}\left(\begin{array}{c}
m \\
0
\end{array}\right) B_{m-0}=B_{m}
$$

From the formulae (12) and (15) we get the primordial relation

$$
\sum_{i=1}^{k+1} \bar{a}_{k, i}\left(n^{2}-n\right)^{i}=\sum_{j=1}^{2 k+1} P_{2 k+1 j}^{-1} n^{j+1}+B_{2 k+1} n=\sum_{j=0}^{2 k+1} \frac{1}{2 k+2}\left(\begin{array}{c}
2 k+2 \\
j+1
\end{array}\right) B_{2 k+1-j} n^{j+1}
$$

Identifying the coefficients of $n^{2 r}$ with $1 \leq r \leq k+1$ and of $n^{2 r+1}, 2 r \leq k$ in both sides of (19) we get the key recursion formulae that are utilizable for obtaining the Faulhaber coefficients as explained hereafter.

$$
\begin{aligned}
& \bar{a}_{k, r}+\left(\begin{array}{c}
r+1 \\
2
\end{array}\right) \bar{a}_{k, r+1}+\ldots+\left(\begin{array}{c}
r+i \\
2 i
\end{array}\right) \bar{a}_{k, r+i}+\ldots+\bar{a}_{k, 2 r}=\sum_{i=0}^{r}\left(\begin{array}{c}
r+i \\
2 i
\end{array}\right) \bar{a}_{k, r+i}=P_{2 k+12 r-1}^{-1} \\
& -\left(\begin{array}{c}
r+1 \\
1
\end{array}\right) \bar{a}_{k, r+1}-\left(\begin{array}{c}
r+2 \\
3
\end{array}\right) \bar{a}_{k, r+2}-\ldots-\left(\begin{array}{c}
2 r+1 \\
2 r+1
\end{array}\right) \bar{a}_{k, 2 r+1}=P_{2 k+12 r}^{-1}=B_{1} \delta_{k 0} \text { or } B_{1} \delta_{k r}
\end{aligned}
$$

3.2 Calculations of the Faulhaber Coefficients $\bar{a}_{k, 1}, \bar{a}_{k, 2}, \bar{a}_{k, 3}, \ldots, \bar{a}_{k, k}, \bar{a}_{k, k+1}$

Thank to the relations (20), (21) we have the following algorithm for calculating them in two stages.

(i) At the first stage, let

$$
\begin{aligned}
& (2 k+1 \quad j)=\left(\begin{array}{c}
2 k+1 \\
j
\end{array}\right) \\
& k^{\prime}=\left[\frac{k+1}{2}\right]
\end{aligned}
$$

we may calculate $\bar{a}_{k, 1}, \bar{a}_{k, 2}, \bar{a}_{k, 3}, \ldots, \bar{a}_{k, k^{\prime}+1}$ from the relations (20) and (21) by the well-structured matrix formula 


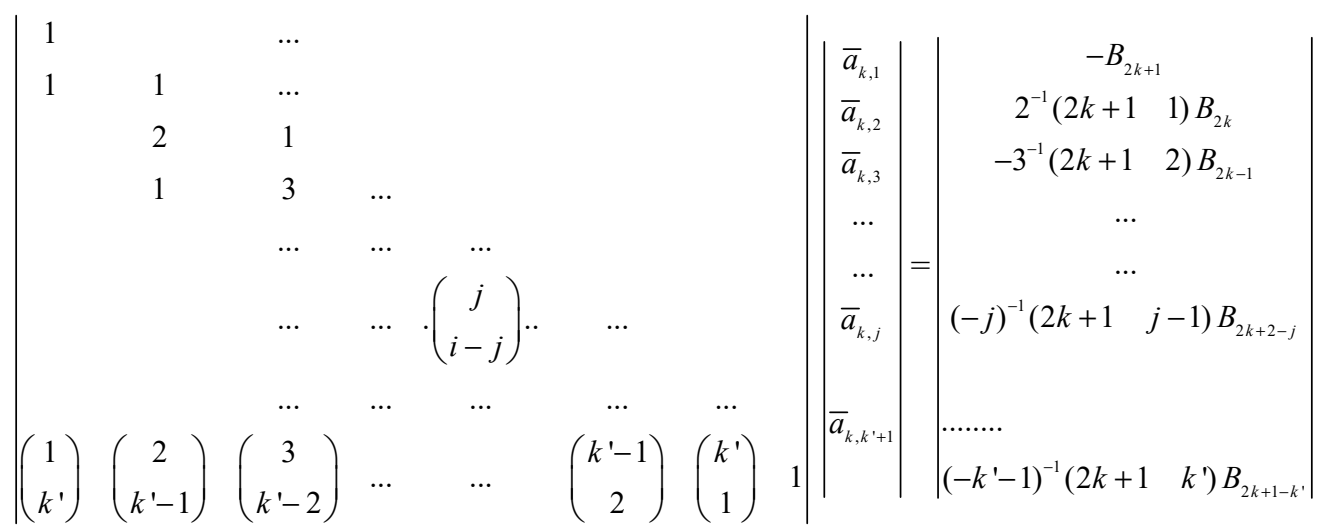

Concretely let $\mathrm{S}$ be the matrix in the left side we may calculate $\bar{a}_{k, j}$ by the formula

$$
\bar{a}_{k, j}=(-)^{j}\left(\begin{array}{c}
2 k+1 \\
j-1
\end{array}\right) B_{2 k+2-j}-S_{j, 1} \bar{a}_{k, 1}-\ldots-S_{j, j-1} \bar{a}_{k, j-1}
$$

for $j=1,2, \ldots, k+1$. Nevertheless it is advantageous to calculate $\bar{a}_{k, j}$ with $j=k^{\prime}+2, \ldots, k+1$ by the following way.

(ii) The second stage of the algorithm consists in utilizing the formula (21) to get successively $\bar{a}_{k, k+1}, \bar{a}_{k, k}, \bar{a}_{k, k-1}, \ldots, \bar{a}_{k, k^{\prime}+2}$ by the matrix formula

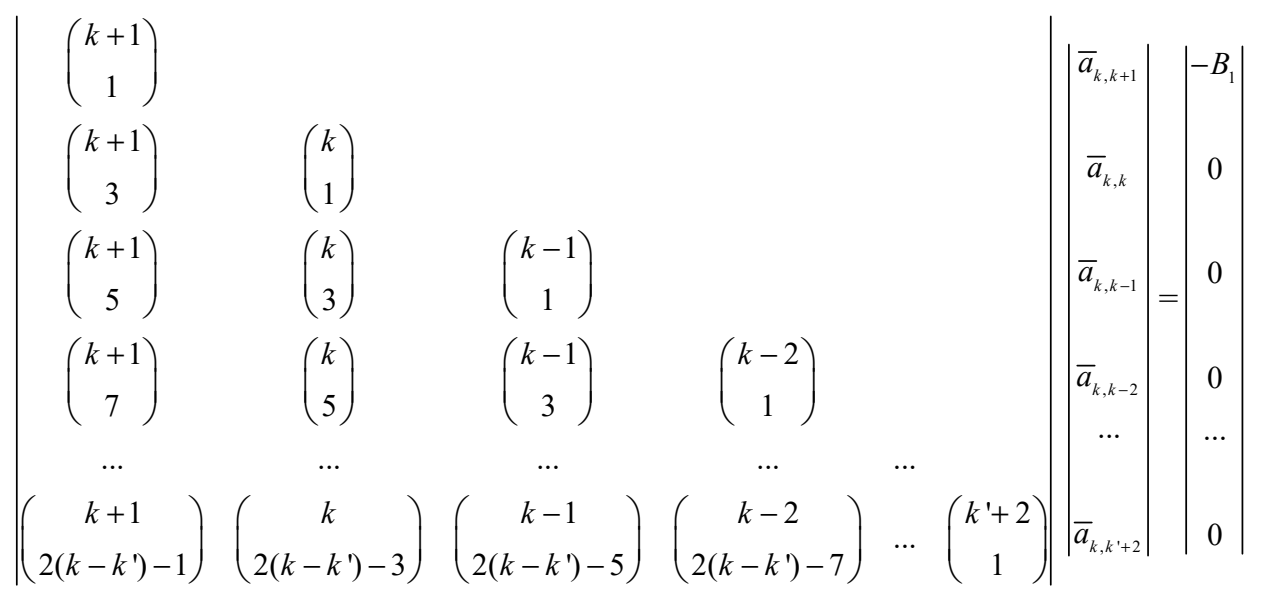

(iii) Examples:

For obtaining $S_{3}(n)$ until $S_{25}(n)$ we utilize the matrix formula (22) to get the equations such as

$$
\left|\begin{array}{l}
\bar{a}_{1,1} \\
\bar{a}_{1,2}
\end{array}\right|=\left|\begin{array}{c}
0 \\
(3 / 2) B_{2}
\end{array}\right|=\left|\begin{array}{c}
0 \\
(1 / 4)
\end{array}\right|
$$

$$
\begin{aligned}
& \text { (...) }
\end{aligned}
$$

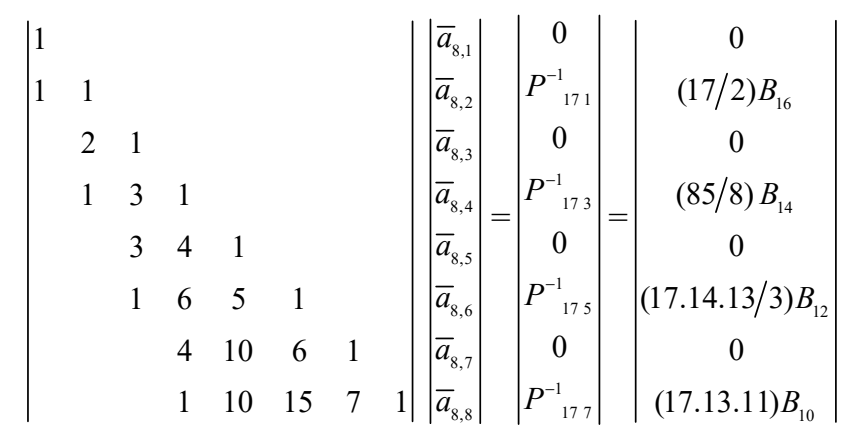

(..) 
and the matrix formula (23) to get such as

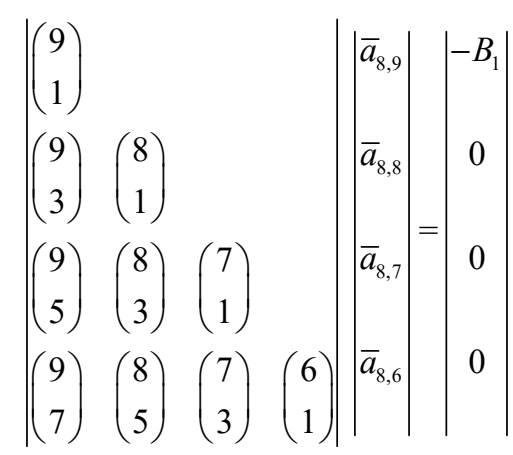

(..)

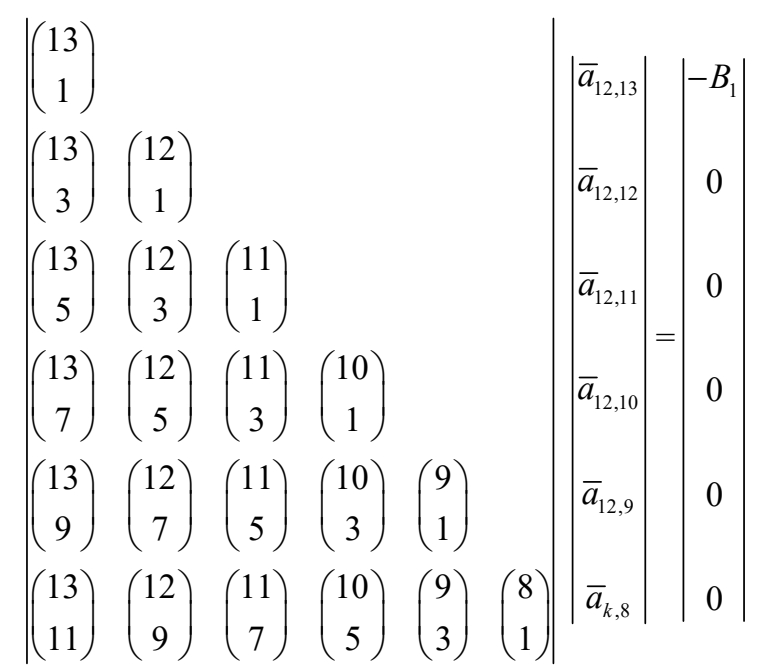

Resolving them we get all the Faulhaber coefficients necessary for calculating the power sums on integers from

$$
S_{3}(n)=S_{1}^{2}(n)
$$

to the well known

$$
\begin{aligned}
S_{17}(n)= & -\frac{3617}{15} S_{1}^{2}(n)+\frac{14468}{15} S_{1}^{3}(n)-\frac{4948}{3} S_{1}^{4}(n)+\frac{24304}{15} S_{1}^{5}(n) \\
& -\frac{9376}{9} S_{1}^{6}(n)+\frac{1408}{3} S_{1}^{7}(n)-\frac{448}{3} S_{1}^{8}(n)+\frac{256}{9} S_{1}^{9}(n)
\end{aligned}
$$

until the maybe waited formula

$$
\begin{aligned}
& S_{25}(n)=\frac{25}{2} B_{24} U^{2}-25 B_{24} U^{3}+\left(\frac{125}{2} B_{24}+575 B_{22}\right) U^{4}-\left(175 B_{24}+2300 B_{22}\right) U^{5} \\
& +\left(525 B_{24}+8050 B_{22}+8855 B_{20}\right) U^{6}-\left(1650 B_{24}+27600 B_{22}+53130 B_{20}\right) U^{7} \\
& -\frac{34526}{3} U^{8}+\frac{9185}{6} U^{9}-\frac{649}{4} U^{10}+\frac{83}{6} U^{11}-\frac{11}{12} U^{12}+\frac{1}{26} U^{13}
\end{aligned}
$$

where

$$
U=\left(n^{2}-n\right)=2 S_{1}(n)
$$

and (Coen, 1996) 


$$
\begin{gathered}
\left\{B_{0}, \ldots . ., B_{17}\right\} \equiv\left\{1,-\frac{1}{2}, \frac{1}{6}, 0,-\frac{1}{30}, 0, \frac{1}{42}, 0,-\frac{1}{30}, 0, \frac{5}{66}, 0, \frac{-691}{2730}, 0, \frac{7}{6}, 0,-\frac{3617}{510}, 0\right\} \\
B_{18}=\frac{43867}{798}, B_{20}=-\frac{174611}{330}, B_{22}=\frac{854513}{138}, B_{24}=-\frac{236364091}{2730}
\end{gathered}
$$

By the way we notice that the preceding results hold for $S_{2 k+1}(n+1)=\sum_{j=0}^{n}(z+j)^{2 k+1}$ because

$$
S_{2 k+1}(n+1)=\sum_{j=1}^{k+1} 2^{-j} a_{k, j}\left((n+1)^{2}-(n+1)\right)^{j}=\sum_{j=1}^{k+1} 2^{-j} a_{k, j}\left(n^{2}+n\right)^{j}=\sum_{j=1}^{k+1} a_{k, j} S_{1}^{j}(n+1)
$$

3. Obtaining the Faulhaber Coefficients Related to $S_{2 k+1}(z, n)$ and $S_{2 k+1}(n)$ from the Function $B_{2 j+1}(z) B_{1}^{-1}(z)$ 3.1 Useful Properties of the Function $B_{2 j+1}(z) B_{1}^{-1}(z)$

(i) Lemma 1:

$$
\text { “ }\left(z^{2}-z\right) B_{l}(z) \text { divides } B_{2 j+1}(z) \text { so that the quotient } B_{2 j+1}(z) B_{1}^{-1}(z) \text { is a polynomial of order } 2 j \text { ” }
$$

Proof: We can find in literature such as in (Do T.S. 2017b) that

$$
B_{2 j+1}(0)=B_{2 j+1}\left(\frac{1}{2}\right)=B_{2 j+1}(1)=0, \quad j>0
$$

and consequently may state that $z(z-1)\left(z-\frac{1}{2}\right)$ divides $B_{2 k+1}(z)$.

QED

In order to calculate the quotient $B_{2 k+1}(z) B_{1}^{-1}(z)$ let

From (31) we get

$$
B_{2 k+1}(z) B_{1}^{-1}(z)=\sum_{1}^{2 k} \beta_{k, j} z^{j}
$$

$$
B_{2 k+1}(z)=\left(z-\frac{1}{2}\right) \sum_{j=1}^{2 k} \beta_{k, j} z^{j}=\sum_{j=1}^{2 k}\left(\beta_{k, j-1}-\frac{1}{2} \beta_{k, j}\right) z^{j}+\beta_{k, 2 k} z^{2 k+1}
$$

On the other hand we have

$$
B_{2 k+1}(z):=(B+z)^{2 k+1}
$$

so that by identification of coefficients we get the recurrence formula for calculating $\beta_{k, j}$

$$
\beta_{k, 0}=0, \ldots, \beta_{k, j}=2 \beta_{k, j-1}-2\left(\begin{array}{c}
2 k+1 \\
j
\end{array}\right) B_{2 k+1-j}, \ldots, \beta_{k, 2 k}=1 .
$$

It is easy to obtain $\beta_{k, j}$ by utilizing MS Excel and the tabulated values of $B_{n}$ in (28).

In particular $\beta_{k, 1}=-2(2 k+1) B_{2 k}, \beta_{k, 2 j}=\beta_{k, 2 j-1}$ excepted the case $\beta_{k, 2 k}=1, \beta_{k, 2 k-1}=-k$.

For example we need to calculate only, $\beta_{8,3}=4 \beta_{8,1}-2\left(\begin{array}{c}17 \\ 3\end{array}\right) B_{14}, \quad \beta_{8,5}=4 \beta_{8,3}-2\left(\begin{array}{c}17 \\ 5\end{array}\right) B_{12}$,

$\beta_{8,7}=4 \beta_{8,5}-2\left(\begin{array}{c}17 \\ 7\end{array}\right) B_{10}, \quad \beta_{8,11}=4 \beta_{8,9}-2\left(\begin{array}{c}17 \\ 11\end{array}\right) B_{6}, \quad \beta_{8,13}=4 \beta_{8,11}-2\left(\begin{array}{l}17 \\ 13\end{array}\right) B_{4}, \quad \beta_{8,15}=4 \beta_{8,13}-2\left(\begin{array}{c}17 \\ 15\end{array}\right) B_{2}$ in order to get $B_{17}(z) B_{1}^{-1}(z)$.

(ii) Lemma 2:

$$
\text { “ } B_{2 j+1}(z) B_{1}^{-1}(z) \text { and } B_{2 j}(z) \text { are polynomials of order } j \text { in } Z=\left(z^{2}-z\right) \text { ” }
$$

Proof: Let 


$$
Z=\left(z^{2}-z\right)
$$

we have

$$
\begin{gathered}
\rho^{2}=4 B_{1}{ }^{2}(z)=(4 Z+1) \\
D_{Z} \equiv \frac{d}{d Z}=\frac{d z}{d Z} \frac{d}{d z} \equiv \frac{1}{2 B_{1}(z)} \frac{d}{d z} \equiv \frac{-1}{\rho} \frac{d}{d z}
\end{gathered}
$$

From a recently proven recurrence formula for Bernoulli polynomials (Do, 2017b)

$$
B_{m}(z)=B_{1}(z) B_{m-1}(z)-\frac{1}{m} \sum_{j=2}^{m}\left(\begin{array}{c}
m \\
j
\end{array}\right) B_{j} B_{m-j}(z)
$$

we get in particular

$$
B_{2 k+1}(z) B_{1}^{-1}(z)=B_{2 k}(z)-\frac{1}{2 k+1} \sum_{j=1}^{k}\left(\begin{array}{c}
2 k+1 \\
2 j
\end{array}\right) B_{2 j} B_{2 k+1-2 j}(z) B_{1}^{-1}(z)
$$

Utilizing the identity beetween operators (Do, 2016)

$$
\begin{gathered}
{\left[D_{z}, f(\hat{z})\right] \equiv f^{\prime}(\hat{z})} \\
D_{z}+a(\hat{z}) \equiv e^{-\int a(z)} D_{z} e^{\int a(z)} \\
\left(D_{z}+a\right) y=f(z) \Rightarrow y=e^{-\int a(z)} \int e^{\int a(z)} f(z)
\end{gathered}
$$

where $\hat{z}$ denotes the Eckaert operator "multiply by the argument $z$ " we get the relation

$$
\begin{aligned}
(2 k+1) B_{2 k}(z) & =D_{z} B_{2 k+1}(z)=D_{z} B_{1}(\hat{z}) B_{1}^{-1}(z) B_{2 k+1}(z) \\
= & \left(B_{1}(\hat{z}) D_{z}+1\right) B_{2 k+1}(z) B_{1}^{-1}(z)
\end{aligned}
$$

which transforms (39) into

i.e. by (40)

$$
\begin{gathered}
2 k \frac{B_{2 k+1}(z)}{B_{1}(z)}=\left(B_{1}{ }^{2}(z) D_{Z}\right) \frac{B_{2 k+1}(z)}{B_{1}(z)}-\sum_{j=1}^{k}\left(\begin{array}{c}
2 k+1 \\
2 j
\end{array}\right) B_{2 j} \frac{B_{2 k+1}(z)}{B_{1}(z)} \\
\left(-\left(Z+\frac{1}{4}\right) D_{Z}+k\right) \frac{B_{2 k+1}(z)}{B_{1}(z)}=-\frac{1}{2} \sum_{j=1}^{k}\left(\begin{array}{c}
2 k+1 \\
2 j
\end{array}\right) B_{2 j} \frac{B_{2 k+1}(z)}{B_{1}(z)}
\end{gathered}
$$

$$
\left(Z+\frac{1}{4}\right)^{k+1} D_{Z}\left(Z+\frac{1}{4}\right)^{-k} \frac{B_{2 k+1}(z)}{B_{1}(z)}=\frac{1}{2} \sum_{j=1}^{k}\left(\begin{array}{c}
2 k+1 \\
2 j
\end{array}\right) B_{2 j} B_{2 k+1-2 j}(z) B_{1}^{-1}(z)
$$

Eq. (42) allows to calculate $B_{5}(z) B_{1}{ }^{-1}(z)$ from $B_{3}(z) B_{1}{ }^{-1}(z)=\left(z^{2}-z\right)=Z$ as followed

$$
\begin{aligned}
& \left(Z+\frac{1}{4}\right)^{3} D_{Z}\left(Z+\frac{1}{4}\right)^{-2} \frac{B_{5}(z)}{B_{1}(z)}=\frac{1}{2}\left(\begin{array}{l}
5 \\
2
\end{array}\right) \frac{1}{6} Z-\frac{1}{2}\left(\begin{array}{l}
5 \\
4
\end{array}\right) \frac{1}{30}=\frac{5}{6} Z-\frac{1}{12} \\
& \frac{12}{64} \frac{B_{5}(z)}{B_{1}(z)}=\frac{10}{4}\left(Z+\frac{1}{4}\right)^{2} \int\left(\frac{4 Z+1}{(4 Z+1)^{3}}-\frac{14}{4(4 Z+1)^{3}}\right)+C\left(Z+\frac{1}{4}\right)^{2}
\end{aligned}
$$

where the constant $C$ is so that $B_{5}(z) B_{1}^{-1}(z)=0$ for $z=0$. Finally we get

$$
\frac{B_{5}(z)}{B_{1}(z)}=Z\left(Z-\frac{1}{3}\right)
$$

and see that it is a polynomial in $Z$. By recursion and (42) we obtain that $B_{2 k+1}(z) B_{1}^{-1}(z)$ is a polynomial of order $k$ in $Z$. Moreover from the recurrence formula (39) we see that so is the polynomial $B_{2 k}(z)$. QED 
In order to calculate $B_{2 k+1}(z) B_{1}^{-1}(z)$ in terms of $Z$, let us introduce the polynomial $\breve{B}_{2 k+1,1}(Z)$ defined by its property

$$
\breve{B}_{2 k+1,1}(Z)=B_{2 k+1}(z) B_{1}^{-1}(z)=\sum_{j=1}^{2 k} \beta_{k, j} z^{j}=\sum_{j=1}^{k} \zeta_{k, j} Z^{j}=\sum_{j=1}^{k} \zeta_{k, j}\left(z^{2}-z\right)^{j}
$$

Eq. (43) is formally similar to Eq. (19) so that by identification of coefficients of $z, z^{2}, \ldots z^{2 k}$ we get the matrix formula giving $\zeta_{k, j}$ similar to (23)

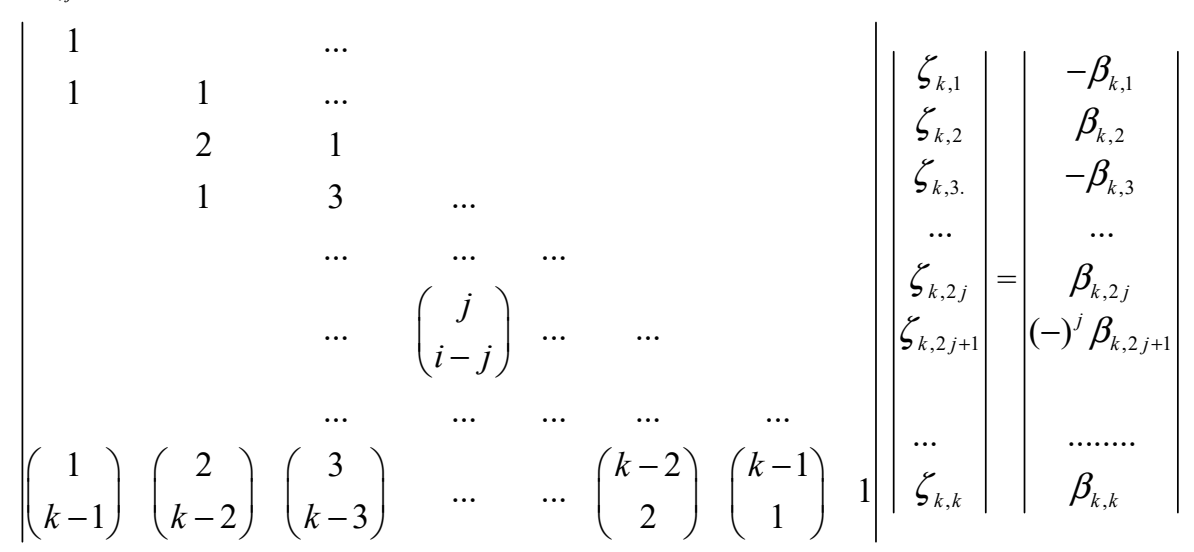

(iii) Algorithm for obtaining $\breve{B}_{2 k+1,1}(Z)$

It consists of inputting in a MS Excel sheet, denoted herein by S, the above matrix elements $\left(\begin{array}{c}j \\ i-j\end{array}\right)$ on $k$ lines, $k$ columns; then $\beta_{k, 1}, \beta_{k, 2}, \ldots, \beta_{k, k}$ on the $(k+1)^{t h}$ line; then utilizing the formula

$$
\zeta_{k, i}=(-)^{i} \beta_{k, i}-\sum_{j=1}^{i-1}\left(\begin{array}{c}
j \\
i-j
\end{array}\right) \zeta_{k, j}
$$

In particular $\zeta_{k, 1}=-\beta_{k, 1}=2(2 k+1) B_{2 k}, \quad \zeta_{k, k}=1$ and, because $\beta_{k, 2 j}=\beta_{k, 2 j-1}, \quad \zeta_{k, 2}=-3 \zeta_{k, 1}$.

Example with $k=8$ :

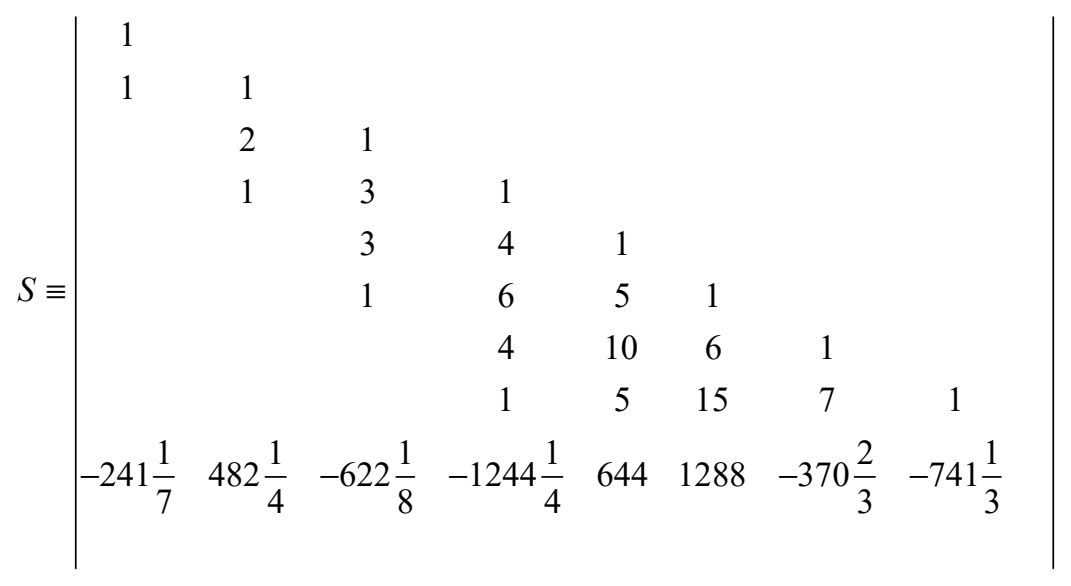

$$
\begin{aligned}
& \zeta_{8,1}=-S_{9,1}=241 \frac{1}{7}, \zeta_{8,2}=-3 \zeta_{8,1}, \quad \zeta_{8,3}=622 \frac{1}{8}-2 \zeta_{8,2}, \quad \zeta_{8,4}=-1244 \frac{1}{4}-\zeta_{8,2}-3 \zeta_{8,3} \\
& \zeta_{8,5}=-644-3 \zeta_{8,3}-4 \zeta_{8,4}, \quad \zeta_{8,6}=1288-\zeta_{8,3}-6 \zeta_{8,4}-5 \zeta_{8,5} \\
& \zeta_{8,7}=370 \frac{2}{3}-4 \zeta_{8,4}-10 \zeta_{8,5}-6 \zeta_{8,6}, \quad \zeta_{8,8}=1
\end{aligned}
$$

We will see later that these values permit to obtain $S_{17}(z, n)$ and $S_{17}(n)$. 


\subsection{Properties of the Faulhaber Coefficients}

(i) Theorem 1

"The Faulhaber coefficients are polynomials in $Z$ "

Proof: Let

$$
\begin{gathered}
\lambda=S_{1}(z, n)=B_{1}(z) n+\frac{1}{2} n^{2}=n z+S_{1}(n)=\frac{1}{2}\left(n^{2}-\rho n\right) \\
S_{2 k+1}(z, n)=\sum_{j=1}^{k+1} a_{k, j}(z) \lambda^{j}=\sum_{j=1}^{k+1} \bar{a}_{k, j}(z)\left(n^{2}-\rho n\right)^{j}
\end{gathered}
$$

where as in (13)

$$
\bar{a}_{k, j}(z)=2^{-j} a_{k, j}(z)
$$

Association of (35) with the Dattoli, Cesarano, Lorenzutta formula (2002)

$$
S_{2 k+1}(z, n)=\sum_{j=0}^{2 k+1} \frac{1}{j+1}\left(\begin{array}{c}
2 k+1 \\
j
\end{array}\right) B_{2 k+1-j}(z) n^{j+1}
$$

leads to the primordial relation

$$
\sum_{j=1}^{k+1} \bar{a}_{k, j}(z)\left(n^{2}-\rho n\right)^{j}=\sum_{j=0}^{2 k+1} \frac{1}{j+1}\left(\begin{array}{c}
2 k+1 \\
j
\end{array}\right) B_{2 k+1-j}(z) n^{j+1}
$$

Comparison of coefficients of $n^{2 r+1}$ in both members of the formula (49) gives

$$
\begin{gathered}
-\left(\begin{array}{c}
r+1 \\
1
\end{array}\right) \rho \bar{a}_{k, r+1}-\left(\begin{array}{c}
r+2 \\
3
\end{array}\right) \rho^{3} \bar{a}_{k, r+2}-\ldots-\left(\begin{array}{c}
2 r+1 \\
2 r+1
\end{array}\right) \rho^{2 r+1} \bar{a}_{k, 2 r+1} \\
=\frac{1}{2 r+1}\left(\begin{array}{c}
2 k+1 \\
2 r
\end{array}\right) B_{2 k+1-2 r}(z) \text { with } r=0,1, \ldots, k
\end{gathered}
$$

Similarly comparison of coefficients of $n^{2 r}$ in both sides of (49) gives

$$
\begin{aligned}
\bar{a}_{k, r} & +\left(\begin{array}{c}
r+1 \\
2
\end{array}\right) \rho^{2} \bar{a}_{k, r+1}+\ldots+\left(\begin{array}{c}
r+i \\
2 i
\end{array}\right) \rho^{2 i} \bar{a}_{k, r+i}+\ldots+\rho^{2 r} \bar{a}_{k, 2 r} \\
& =\frac{1}{2 r}\left(\begin{array}{c}
2 k+1 \\
2 r-1
\end{array}\right) B_{2 k+2-2 r}(z) \quad \text { with } r=1, \ldots, k+1 .
\end{aligned}
$$

In (50) and (51), $\bar{a}_{k, r}$ stands for $\bar{a}_{k, r}(z)$ for saving places.

From (50) we get the very important relation

$$
\bar{a}_{k, 1}(z)=-\frac{1}{\rho} B_{2 k+1}(z)=\frac{1}{2} B_{2 k+1}(z) B_{1}^{-1}(z)=\frac{1}{2} \breve{B}_{2 k+1,1}(Z)
$$

saying that $\bar{a}_{k, 1}(z)$ is a polynomial of order $k$ in $Z$.

Afterward from (51) we get the formula

$$
\bar{a}_{k, 1}(z)+\rho^{2} \bar{a}_{k, 2}(z)=\frac{1}{4}(2 k+1) B_{2 k}(z)
$$

which, because $B_{2 k}(z)$ is a polynomial of order $k$ in $Z$ by (34), shows that $\bar{a}_{k, 2}(z)$ is a polynomial of order $(k-1)$ in $Z$.

Repeating these operations step by step we obtain that $\bar{a}_{k, j}(z)$ is a polynomial of $\operatorname{order}(k-j+1)$ in $Z$. QED

Following this result we may denote from now on a Faulhaber coefficient by

$$
\alpha_{k, j}(Z)=a_{k, j}(z(Z))
$$


(ii). Theorem 2

“The Faulhaber coefficients $\alpha_{k, j}(Z)$ may be calculated from $\alpha_{k, 1}(Z)$

by the formula

$$
\alpha_{k, j}(Z)=\frac{2^{j-1}}{j !} \alpha_{k, 1}^{(j-1)}(Z)=\frac{2^{j-1}}{j !} \breve{B}_{2 k+1,1}^{(j-1)}(Z)
$$

so that the power sums on an arithmetic progression has the form

$$
S_{2 k+1}(z, n)=\sum_{j=1}^{k+1} \alpha_{k, j}(Z) \lambda^{j}=\sum_{j=1}^{k+1} \frac{2^{j-1}}{j !} \breve{B}_{2 k+1,1}^{(j-1)}(Z) S_{1}^{j}(z, n)
$$

where by (43)

$$
\breve{B}_{2 k+1,1}^{(j-1)}(Z)=\sum_{i=0}^{k-j+1} \zeta_{k, i+j-1} \frac{(i+j-1) !}{i !} Z^{i},
$$

Proof: We may write (50), (51) under the form

$$
\begin{gathered}
\bar{\alpha}_{k, r}+\left(\begin{array}{c}
r+1 \\
2
\end{array}\right) \rho^{2} \bar{\alpha}_{k, r+1}+\ldots+\left(\begin{array}{c}
r+i \\
2 i
\end{array}\right) \rho^{2 i} \bar{\alpha}_{k, r+i}+\ldots+\rho^{2 r} \bar{a}_{k, 2 r}=\frac{1}{(2 r) !} D_{z}^{2 r-1} B_{2 k+1}(z) \\
-\left(\begin{array}{c}
r+1 \\
1
\end{array}\right) \bar{\alpha}_{k, r+1}-\left(\begin{array}{c}
r+2 \\
3
\end{array}\right) \rho^{2} \bar{\alpha}_{k, r+2}-\ldots-\left(\begin{array}{c}
2 r+1 \\
2 r+1
\end{array}\right) \rho^{2 r} \bar{\alpha}_{k, 2 r+1}=\frac{1}{(2 r+1) ! \rho} D_{z}^{2 r} B_{2 k+1}(z)
\end{gathered}
$$

where $\bar{\alpha}_{k, r}$ stands for $\bar{\alpha}_{k, r}(Z)$ for saving places.

Derivation with respect to $Z$ the equation (57) gives

$$
\begin{gathered}
D_{Z}\left(\bar{\alpha}_{k, r}+\ldots+\left(\begin{array}{c}
r+i-1 \\
2 i-2
\end{array}\right) \rho^{2 i-2} \bar{\alpha}_{k, r+i-1}+\left(\begin{array}{c}
r+i \\
2 i
\end{array}\right) \rho^{2 i} \bar{\alpha}_{k, r+i}+\ldots+\rho^{2 r} \bar{\alpha}_{k, 2 r}\right) \\
=\frac{-1}{(2 r) ! \rho} D_{z}^{2 r} B_{2 k+1}(z)
\end{gathered}
$$

With the hypothesis

$$
\bar{\alpha}_{k, j+1}(Z)=(j+1)^{-1} \bar{\alpha}_{k, j}^{\prime}(Z) \forall j=1,2, \ldots, 2 r-1
$$

and the property

$$
D_{Z} \rho^{2 j}=\frac{d \rho^{2}}{d Z} \frac{d}{d \rho^{2}} \rho^{2 j}=\frac{d(4 Z+1)}{d Z} \frac{d}{d \rho^{2}}\left(\rho^{2}\right)^{j}=4 j \rho^{2 j-2}
$$

we may write instead of (59)

$$
\begin{gathered}
(r+1) \bar{\alpha}_{k, r+1}+4\left(\begin{array}{c}
r+1 \\
2
\end{array}\right) \bar{\alpha}_{k, r+1}+\ldots+\left(\begin{array}{c}
r+i-1 \\
2 i-2
\end{array}\right) \rho^{2 i-2}(r+i) \bar{\alpha}_{k, r+i}+\left(\begin{array}{c}
r+i \\
2 i
\end{array}\right) 4 i \rho^{2 i-2} \bar{\alpha}_{k, r+i} \\
+\ldots+4 r \rho^{2 r-2} \bar{\alpha}_{k, 2 r}+\rho^{2 r} \bar{\alpha}_{k, 2 r}^{\prime}=\frac{-1}{\rho(2 r) !} D_{z}^{2 r} B_{2 k+1}(z)
\end{gathered}
$$

In the above formula the coefficient of $\bar{\alpha}_{k, r+i}(Z)$ is

$$
\left(\begin{array}{c}
r+i-1 \\
2 i-2
\end{array}\right) \rho^{2 i-2}(r+i)+\left(\begin{array}{c}
r+i \\
2 i
\end{array}\right) 4 i \rho^{2 i-2}=(2 r+1)\left(\begin{array}{c}
r+i \\
2 i-1
\end{array}\right) \rho^{2 i-2}
$$

On the other hand multiplying (58) by $(2 r+1)$ then comparing the result with (61) and taking account on (62) we get the relation

$$
\bar{\alpha}_{k, 2 r}^{\prime}(Z)=(2 r+1) \bar{\alpha}_{k, 2 r+1}(Z)
$$

saying that the hypothesis (60) is right for $\forall j=1,2, \ldots, 2 r$ 
Differentiation with respect to $Z$ the equation (58) and utilization of the preceding argumentations lead to the conclusion

$$
\bar{\alpha}_{k, j}^{\prime}(Z)=(j+1) \bar{\alpha}_{k, j+1}(Z) \quad \forall j \geq 1
$$

QED

Examples:

$$
\begin{aligned}
& \quad k=1 \\
& \bar{\alpha}_{1,1}(z)=\frac{1}{2} \frac{B_{3}(z)}{B_{1}(z)}=\frac{1}{2} Z, \quad \bar{\alpha}_{1,2}(z)=\frac{1}{2} \bar{\alpha}_{1,1}^{\prime}(z)=\frac{1}{4} \\
& S_{3}(z, n)=\sum_{j=1}^{2} \alpha_{1, j}(z) \lambda^{j}=Z \lambda+\lambda^{2} \\
& \quad k=2 \\
& \bar{\alpha}_{2,1}(Z)=\frac{1}{2} \frac{B_{5}(z)}{B_{1}(z)}=\frac{1}{2}\left(Z^{2}-\frac{1}{3} Z\right), \quad \bar{\alpha}_{2,2}(Z)=\frac{1}{2}\left(Z-\frac{1}{6}\right), \quad \bar{\alpha}_{2,3}(Z)=\frac{1}{3} \frac{1}{2} \\
& S_{5}(z, n)=\left(Z^{2}-\frac{1}{3} Z\right) \lambda+\left(2 Z-\frac{1}{3}\right) \lambda^{2}+\frac{4}{3} \lambda^{3}
\end{aligned}
$$

(iii). Theorem 3

"The power sums of integers may be calculated from $\breve{B}_{2 k+1,1}(Z)$ by

the formula

$$
S_{2 k+1}(n)=\sum_{j=2}^{k+1} \frac{2^{j-1}}{j !} \breve{B}_{2 k+1,1}{ }^{(j-1)}(0) S_{1}^{j}(n)=\sum_{j=2}^{k+1} \frac{2^{j-1}}{j} \zeta_{k, j-1} S_{1}^{j}(n) ”
$$

Proof: From (43) we get

$$
\breve{B}_{2 k+1,1}^{(j-1)}(0)=(j-1) ! \zeta_{k, j-1}
$$

so that formula (56) is reduced to (64). QED

For example we may calculate $S_{17}(n)$ by the formula

$$
\begin{aligned}
S_{17}(n)=\zeta_{8,1} S_{1}^{2}(n) & +\frac{4}{3} \zeta_{8,2} S_{1}^{3}(n)+\frac{8}{4} \zeta_{8,3} S_{1}^{3}(n)+\frac{16}{5} \zeta_{8,4} S_{1}^{4}(n)+\frac{32}{6} \zeta_{8,5} S_{1}^{5}(n) \\
+ & \frac{64}{7} \zeta_{8,6} S_{1}^{6}(n)+\frac{128}{8} \zeta_{8,7} S_{1}^{7}(n)+\frac{128}{8} S_{1}^{8}(n)
\end{aligned}
$$

with $\zeta_{8, k}$ given herein before.

From (64) we see that the power sums on integers may be written under the vectorial form

$$
S_{2 k+1}(n)=\vec{C} \vec{B}_{2 k++1,1} \vec{S}_{1}(n)
$$

where we define

$$
\begin{gathered}
\vec{C} \equiv\left\{\begin{array}{llllllll}
\frac{1}{1} & \frac{2}{2} & \frac{4}{3} & \frac{8}{4} & \frac{16}{5} & \frac{32}{6} & \frac{64}{7} & \ldots
\end{array}\right\} \\
\overrightarrow{\vec{B}}_{2 k+1,1} \equiv\left\{\begin{array}{llllllll}
\zeta_{k, 0}=0 & \zeta_{k, 1} & \zeta_{k, 2} & \zeta_{k, 3} & \zeta_{k, 4} & \zeta_{k, 5} & \zeta_{k, 6} & \ldots
\end{array}\right\} \\
\vec{S}_{1}(n) \equiv\left\{\begin{array}{llllllll}
S_{1}(n) & S_{1}^{2}(n) & S_{1}^{3}(n) & S_{1}^{4}(n) & S_{1}^{5}(n) & S_{1}^{6}(n) & S_{1}^{7}(n) & \ldots
\end{array}\right\}
\end{gathered}
$$

and adopt a whynot generalized scalar product between three vectors.

For example 


$$
\begin{gathered}
\breve{B}_{7,1}(Z)=\frac{1}{3} Z-Z^{2}+Z^{3} \\
S_{7}(n)=\frac{1}{3} S_{1}{ }^{2}(n)-\frac{4}{3} S_{1}^{3}(n)+2 S_{1}^{4}(n)
\end{gathered}
$$

\section{Obtaining the Power Sums $S_{2 k+1}(z, n)$ From $S_{2 k+1}(n)$}

\subsection{Theorem 4}

"All the Faulhaber coeficients $\bar{\alpha}_{k, j}(Z)$ may successively be calculated by

the formula

$$
\bar{\alpha}_{k, j}(Z)=(j+1) \int_{0}^{Z} \bar{\alpha}_{k, j+1}(Z) d Z+\bar{\alpha}_{k, j}(0)
$$

beginning from the last of them which is $\bar{\alpha}_{k, k+1}(Z)=\frac{1}{2 k+2}$ "

Proof: By integrating both members of the formula coming from (60)

$$
\bar{\alpha}_{k, j}^{\prime}(Z)=(j+1) \bar{\alpha}_{k, j+1}(Z) \quad \forall j \geq 1
$$

and taking the right constant of integration. QED

\subsection{Theorem 5}

“ The $j^{\text {th }}$ Faulhaber coefficient in a sum of powers on an arithmetic progression

is equal to $\frac{2^{j}}{j !}$ times the $j^{\text {th }}$ derivative of the corresponding sum of powers of integers expressed in term of

the argument $Z=z(z-1)$ instead of $U=n(n-1)$. “

Proof: According to (64), by integrating $k$ times the constant coefficient $\bar{a}_{k, k+1}$ with respect to $Z$ we will get $\bar{\alpha}_{k, 1}(Z)$ of which

○ the term containing $Z^{k}$ is $(k+1) ! \bar{a}_{k, k+1} \frac{Z^{k}}{k !}=(k+1) \bar{a}_{k, k+1} Z^{k}$

○ ...

○ the term containing $Z^{j}$ is $(j+1) ! \bar{a}_{k, j+1} \frac{Z^{j}}{j !}=(j+1) \bar{a}_{k, j+1} Z^{j}$

that is to say

$$
\bar{\alpha}_{k, 1}(Z)=\sum_{j=1}^{k}(j+1) \bar{a}_{k, j+1} Z^{j}
$$

This relation is to be compared with the relation coming from (52)

$$
\alpha_{k, 1}(Z)=\breve{B}_{2 k+1,1}(Z)=\sum_{j=1}^{k} \zeta_{k, j} Z^{j}
$$

Introducing the function

$$
\hat{S}_{2 k+1}(Z) \equiv S_{2 k+1}(z)=\sum_{j=0}^{k} \bar{a}_{k, j+1}\left(z^{2}-z\right)^{j+1}=\sum_{j=0}^{k} \bar{a}_{k, j+1} Z^{j+1}
$$

we have curiously by comparing (71), (72) the important relation

$$
\bar{\alpha}_{k, 1}(Z)=\hat{S}_{2 k+1}^{\prime}(Z)=\frac{1}{2} \breve{B}_{2 k+1,1}(Z)
$$

which because

$$
\bar{\alpha}_{k, j}(Z)=\frac{1}{j} \bar{\alpha}_{k, j-1}^{\prime}(Z)=\ldots=\frac{1}{j !} \bar{\alpha}_{k, 1}{ }^{(j-1)}(Z)=\frac{1}{j !} \hat{S}_{2 k+1}{ }^{(j)}(Z)
$$


gives rise to the ultimate formula linking $S_{2 k+1}(z, n)$ with $S_{2 k+1}(n)$

or under symbolical (umbral) form

$$
S_{2 k+1}(z, n)=\sum_{j=1}^{k+1} \frac{2^{j}}{j !} \hat{S}_{2 k+1}^{(j)}(Z) S_{1}^{j}(z, n)
$$

$$
S_{2 k+1}(z, n):=\exp 2 \hat{S}_{2 k+1}{ }^{(\cdot)}(Z) S_{1}(z, n)-1 \quad \text { QED }
$$

For examples

$$
\begin{aligned}
& \circ S_{3}(n)=\left(\frac{n(n-1)}{2}\right)^{2} \Rightarrow \hat{S}_{3}(Z)=S_{3}(z)=\frac{1}{4} Z^{2} \\
& S_{3}(z, n)=\frac{2^{1}}{1}\left(\frac{1}{4} Z^{2}\right)^{\prime} \lambda+\frac{2^{2}}{2}\left(\frac{1}{4} Z^{2}\right)^{\prime \prime} \lambda^{2}=Z \lambda+\lambda^{2}
\end{aligned}
$$

○ From (25) we have

$$
\begin{aligned}
\hat{S}_{17}(Z)= & -\frac{3617}{15} \frac{Z^{2}}{2^{2}}+\frac{14468}{15} \frac{Z^{3}}{2^{3}}-\frac{4948}{3} \frac{Z^{4}}{2^{4}}+\frac{24304}{15} \frac{Z^{5}}{2^{5}} \\
& -\frac{9376}{9} \frac{Z^{6}}{2^{6}}+\frac{1408}{3} \frac{Z^{7}}{2^{7}}-\frac{448}{3} \frac{Z^{8}}{2^{8}}+\frac{256}{9} \frac{Z^{9}}{2^{9}}
\end{aligned}
$$

5. Obtaining $S_{2 k}(z, n)$ from $S_{2 k+1}(z, n)$

\subsection{Theorem 6:}

"The sums of even powers on arithmetic progressions may be calculated from the formula

$$
(2 k+1) S_{2 k}(z, n)=2 \hat{S}_{2 k+1}^{\prime}(Z) n+(2 n+2 z-1) \sum_{j=1}^{k} \frac{2^{j}}{j !} \hat{S}_{2 k+1}^{(j+1)}(Z) \lambda^{j} ”
$$

Proof: From literature for example in (Do T.S. 2017b) we know that $S_{m}{ }^{\prime}(z, n)=m S_{m-1}(z, n)$

This formula and (37), (75) and the fact that

give rise to the formula

$$
\lambda^{\prime}=S_{1}^{\prime}(z, n)=\left(B_{1}(z) n+\frac{n^{2}}{2}\right)^{\prime}=n B_{1}^{\prime}(z)=n
$$

$$
(2 k+1) S_{2 k}(z, n)=S_{2 k+1}^{\prime}(z, n)=\sum_{j=1}^{k+1} \frac{2^{j}}{j !} \hat{S}_{2 k+1}{ }^{(j)}(Z) n j \lambda^{j-1}+\frac{2^{j}}{j !} 2 B_{1}(z) \hat{S}_{2 k+1}{ }^{(j+1)}(Z) \lambda^{j}
$$

which clearly leads to the formula (78). QED

\subsection{Examples}

$$
\begin{aligned}
& \hat{S}_{3}(Z)=S_{3}(z)=S_{1}^{2}(z)=\frac{Z^{2}}{4} \\
& 3 S_{2}(z, n)=Z n+(2 z+2 n-1) \frac{2}{1 !}\left(\frac{Z^{2}}{4}\right) " \lambda=Z n+(2 z+2 n-1) \lambda \\
& \circ \quad \hat{S}_{5}(Z)=-\frac{1}{12} Z^{2}+\frac{1}{6} Z^{3} \\
& 5 S_{4}(z, n)=S_{5}^{\prime}(z, n)=\left(Z^{2}-\frac{1}{3} Z\right) n+(2 z+2 n-1)\left(\left(2 Z-\frac{1}{3}\right) \lambda+2 \lambda^{2}\right)
\end{aligned}
$$

\section{Remarks and Conclusions}

In a precedent work (Do T. S. 2017b) we have proven thank to translation operators the property

$$
S_{m}(z, n)=z^{m}+(z+1)^{m}+\ldots+(z+n-1)^{m}=\frac{e^{n D_{z}}-1}{e^{D_{z}}-1} z^{m}
$$


and the formula linking it with the Bernoulli polynomials $B_{m}(z) \equiv \frac{D_{z}}{e^{D_{x}}-1} z^{m}$

$$
(m+1) S_{m}(z, n)=\sum_{k=1}^{m+1}\left(\begin{array}{c}
m+1 \\
k
\end{array}\right) B_{m-k+1}(z) n^{k}:=(B(z)+n)^{m+1}-B^{m+1}(z) .
$$

This formula contains the Dattoli, Cesarano, Lorenzutta (2002) formula which we may put under the form

$$
S_{m+1}(z, n)=\int_{0}^{n} B_{m}(z+t) d t:=\int_{0}^{n}(B+z+t)^{m} d t
$$

In the present work we firstly resume the theorem of Chen, Fu, Zhang (2009) proving the Faulhaber conjecture from the preceding formula; secondly compare the Faulhaber formula on power sums of integers with the Pascal formula (Do, 2017a)

$$
S_{2 k+1}(n)=\sum_{i=1}^{k+1} \bar{a}_{k, i}\left(n^{2}-n\right)^{i}=\sum_{j=1}^{2 k+1} P_{2 k+1 j}^{-1} n^{j+1}+B_{2 k+1} n
$$

for obtaining the formula giving the Faulhaber coefficients, for examples in $S_{17}(n), S_{25}(n)$.

Thirdly we compare the conjectured Faulhaber formula on powers sums $S_{2 k+1}(z, n)$ with the Dattoli, Cesarano, Lorenzutta formula

$$
S_{2 k+1}(z, n)=\sum_{j=1}^{k+1} a_{k, j}(z) S_{1}^{j}(z, n)=\sum_{j=0}^{2 k+1} \frac{1}{j+1}\left(\begin{array}{c}
2 k+1 \\
j
\end{array}\right) B_{2 k+1-j}(z) n^{j+1}
$$

and obtain two equations for calculating the set of Faulhaber coefficients $a_{k, i}(z)$. Managing these equations , firstly we prove that the function $B_{2 k+1}(z) B_{1}^{-1}(z)$ is a polynomial in $Z=z(z-1)$ easily calculable, denoted herein by $\bar{B}_{2 k+1,1}(Z)$; secondly that all the Faulhaber coefficients $a_{k, i}(z)$ are also polynomials in $Z$, denoted consequently by $\alpha_{k, j}(Z)$; thirstly that all the $\alpha_{k, j}(Z)$ are calculable by derivation from the first of them which is equal to $\breve{B}_{2 k+1,1}(Z)$. At this stage we get the Faulhaber expansion

$$
S_{2 k+1}(z, n)=\sum_{j=1}^{k+1} \frac{2^{j-1}}{j !} \breve{B}_{2 k+1,1}{ }^{(j-1)}(Z) S_{1}^{j}(z, n)
$$

Afterward we introduce the functions $\hat{S}_{2 k+1}(Z) \equiv S_{2 k+1}(z)$ from the power sums on integers $S_{2 k+1}(n)$ and arrive to obtain another form of Faulhaber expansion

which leads to

$$
S_{2 k+1}(z, n)=\sum_{j=1}^{k+1} \frac{2^{j}}{j !} \hat{S}_{2 k+1}^{(j)}(Z) S_{1}^{j}(z, n)
$$

$$
(2 k+1) S_{2 k}(z, n)=2 \hat{S}^{\prime}{ }_{2 k+1}(Z) n+(2 n+2 z-1) \sum_{j=1}^{k} \frac{2^{j}}{j !} \hat{S}_{2 k+1}{ }^{(j+1)}(Z) \lambda^{j}
$$

By comparing these two forms of Faulhaber expansion we get the beautiful formulae

$$
\begin{aligned}
& 2 \hat{S}_{2 k+1}(Z)=\int_{0}^{Z} \breve{B}_{2 k+1,1}(t) d t \\
& 2 S_{2 k+1}(n)=\int_{0}^{n(n-1)} \breve{B}_{2 k+1,1}(t) d t=\int_{0}^{2 S_{1}(n)} \breve{B}_{2 k+1,1}(t) d t
\end{aligned}
$$

On behalf of the results obtained recently by Edwards (1986), Knuth (1993), Chen, Fu, and Zhang (2009) and the results obtained in this work, we may claim that the Faulhaber problem on power sums are now quasi if not fully resolved.

A bemol subsists however because herein the proof for $B_{2 k+1}(z) B_{1}^{-1}(z)$ to be a polynomial in $\mathrm{Z}$ seems cumbersome. Subsists also the challenge of extending the results of this work to sums of real power on arithmetic progressions as preconized Knuth (1993) for power sums of integers.

The author dedicate this work to his adorable wife for the vietnamese scented tea she silently offered him from time to time during the long days he performed this work. 


\section{References}

Beardon, A. F. (1996). Sums of powers of integers. Amer. Math. Monthly, 103, 201-213.

Beery, J. (2010). Sums of Powers of Positive Integers - Introduction. Mathe matical Association of America. Retrieved from http://www.maa.org/press/periodicals/convergence/sums-of-powers-of-positive-integersintroduction

Bernoulli, J. (1713). Ars Conjectandi. was published posthumously in Euler L., 1738.

Chen, W. Y., Fu, A. M., \& Zhang, I. F. (2009). Faulhaber's theorem on power sums. Discrete Mathematics, 309(10), 2974-2981.

Coen, L. E. S. (1996). Sums of powers and the Bernoulli numbers (Master's thesis, Eastern Illinois University). Retrieved from http://thekeep.eiu.edu/theses/1896

Dattoli, G. I. U. S. E. P. P. E., Lorenzutta, S., \& Cesarano, C. (2002). Bernoulli numbers and polynomials from a more general point of view. Rend. Mat. Appl, 22(7), 193-202.

Do, T. S. (2016). Operator calculus. Edification and Utilization. Lambert Academic Publishing.

Do, T. S. (2017a). Sums of powers of integers and Bernoulli numbers clarified. Applied Physics Research, 9(2), 12-20. http://dx.doi.org/10.5539/apr.v9n2p12

Do, T. S. (2017b). Obtaining Easily Sums of Powers on Arithmetic Progressions and Properties of Bernoulli Polynomials by Operator Calculus. Applied Physics Research, 9(5), 158-187. https://doi.org/10.5539/ apr.v9n5p73

Edwards, A. W. F. (1986). A quick route to sums of powers. The American Mathematical Monthly, 93(6), 451-455.

Euler L. (1738). Methodus generalis summandi progressiones. Comment. Acad. Sci. Petrop., 6.

Faulhaber, J. (1631). Academia Algebrce, Darinnen die miraculosische Inventiones zu den höchsten Cossen weiters continuirt und profitiert warden. Augsburg, bey Johann Ulrich Schönigs.

Ireland, K., \& Rosen, M. (2013). A classical introduction to modern number theory (Vol. 84). Springer Science $\&$ Business Media.

Jacobi, C. G. J. (1834). De usu legitimo formulae summatoriae Maclaurinianae. J. Reine Angew. Math., 12, 263-72.

Johnson, J. A. (1986). Summing the powers of the integers using calculus. The Mathematics Teacher, 79(3), 218-219.

Knuth, D. E. (1993). Johann Faulhaber and sums of powers. Mathematics of Computation, 61(203), 277-294.

Tits, L. (1923). Sur la sommation des puissances numériques. Mathesis, 37, 353-355.

\section{Copyrights}

Copyright for this article is retained by the author(s), with first publication rights granted to the journal.

This is an open-access article distributed under the terms and conditions of the Creative Commons Attribution license (http://creativecommons.org/licenses/by/4.0/). 